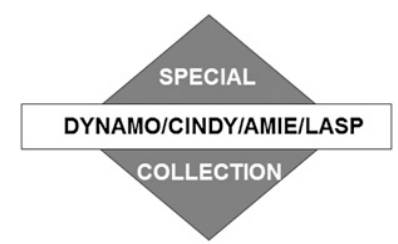

\title{
๖The Diurnal and Microphysical Characteristics of MJO Rain Events during DYNAMO
}

\author{
ANGELA K. Rowe \\ University of Washington, Seattle, Washington \\ ROBERT A. HOUZE JR. \\ University of Washington, Seattle, and Pacific Northwest National Laboratory, Richland, Washington \\ STACY BRODZIK \\ University of Washington, Seattle, Washington \\ MANUEL D. ZULUAGA \\ Climate Forecast Application Network, Reno, Nevada, and Universidad Nacional de Colombia, Medellín, Colombia
}

(Manuscript received 19 October 2018, in final form 10 April 2019)

\begin{abstract}
The Madden-Julian oscillation (MJO) dominates the intraseasonal variability of cloud populations of the tropical Indian and Pacific Oceans. Suppressed MJO periods consist primarily of shallow and isolated deep convection. During the transition to an active MJO, the shallow and isolated deep clouds grow upscale into the overnight hours. During active MJO periods, mesoscale convective systems occur mostly during 2-4-day bursts of rainfall activity with a statistically significant early morning peak. Yet when these rain events are separated into individual active periods, some periods do not follow the mean pattern, with the November events in particular exhibiting an afternoon peak. The radar-observed microphysical processes producing the precipitation during the major rain events of active MJO periods evolve in connection with synoptic-scale wave passages with varying influences of diurnal forcing. MJO studies that do not account for the intermittency of rainfall during active MJO phases through averaging over multiple events can lead to the misimpression that the primary rain-producing clouds of the MJO are modulated solely by the diurnal cycle.
\end{abstract}

\section{Introduction}

The Madden-Julian oscillation (MJO) is a mode of a 30-90-day period that divides broadly into a "suppressed period" and an "active period" (Madden and Julian 1971, 1972). During the MJO suppressed period over the tropical Indian Ocean, clouds are primarily shallow, organized into lines or open cells (Rowe and Houze 2015), with occasional precipitating convection forming during the afternoon. As the suppressed period transitions toward the active MJO period, increasingly

D Denotes content that is immediately available upon publication as open access.

Corresponding author: Angela Rowe, akrowe@uw.edu deeper and more spatially organized convection forms along intersecting cold pool boundaries (Rowe and Houze 2015; Feng et al. 2015), eventually growing upscale into mesoscale convective systems (MCSs) in the overnight hours. Within an active MJO period, major "rain events" (REs) occur in 2-4-day bursts, during which deep MCSs are associated with synoptic-scale wave passages (e.g., Zuluaga and Houze 2013, hereafter ZH13; Powell and Houze 2013). During these REs, shallow convection transitions to deep convection under unstable conditions, eventually growing to wide convective entities as rainfall and large-scale vertical motion maximizes with pronounced low-level convergence, and then into broad stratiform regions with periods of embedded convection (ZH13). Periods of relatively weaker convection, in terms of rainfall, separate these 
REs, referred to as "nonevent" (NE) periods, within active MJO periods.

Using data from the Dynamics of the MJO (DYNAMO) field campaign (Yoneyama et al. 2013), Sakaeda et al. (2018) analyzed the diurnal cycle of rainfall in suppressed and active MJO periods. However, their analysis does not separate the 2-4-day REs from the relatively quieter NE periods separating the REs. In this paper, we make this separation and examine the question of whether the diurnal cycle continues to modulate convection during the 2-4-day REs that develop after the shallow-to-deep convective transition. This question is critical because most of the MJO rainfall occurs in the REs, and it is important to know whether or not diurnal radiative processes (e.g., Randall et al. 1991; Liu and Moncrieff 1998) play a role in the physical production of MJO rainfall.

In this study, we explore the answer to this question by

1) determining if the gross structures of radar echoes seen by the National Center for Atmospheric Research (NCAR) S-band/Ka-band (S-PolKa) deployed during DYNAMO show a statistical indication of diurnal variability during 2-4-day REs and

2) using the dual-polarization capability of S-PolKa to determine whether the microphysical characteristics of the precipitation particles evolve in ways that are related to diurnal effects on the precipitation mechanisms at play in the MJO REs.

It is important to note that it is possible for the diurnal cycle to affect the timing of the deep convective rain episodes on a 2-day time scale. Chen and Houze (1997) showed how the upper-level cloud shield of a mesoscale deep convective system over the warm tropical ocean shades the ocean and prevents the surface air temperature from recovering for a day after a deep convective episode. The result is a bidiurnal cycle of deep convection at a specific location. Sometimes this bidiurnal cycle can become sympathetic with a 2-day propagating wave. Yu et al. (2018) found evidence that 2-day rainfall variability was associated with the relationship between the diurnal cycle and quasi-2-day westward-propagating disturbances during the October MJO event in DYNAMO. Their result differs from our objective in that we are not concerned with the processes that determine the exact timing of the bursts of deep convective activity manifested as REs during an active MJO period, but rather with whether diurnal variability affects the precipitation mechanisms during an RE once it occurs.

Our data sources and methods are described in section 2. Section 3 explores the diurnal cycle of the major types of radar echo within the context of MJO phases and the REs. Section 4 examines the time variation of the microphysical processes of convection in relation to diurnal and synoptic controls. In section 5, we integrate the conclusions and implications of these results.

\section{Instrumentation, data, and methods}

\section{a. S-PolKa}

The NCAR dual-polarization, dual-wavelength, Doppler S-PolKa radar was deployed on the Addu Atoll from 1 October 2011 through 15 January 2012. S-PolKa's 15-min scanning strategy featured $360^{\circ}$ azimuthal surveillance (SUR) scans at eight constant elevation angles from $0.5^{\circ}$ to $11.0^{\circ}$ followed by two sectors of range-height indicator (RHI) scans directed over the ocean up to $45^{\circ}$ elevation. For this study, we focus on the SUR scans to capture the full areal extent of systems in S-PolKa's domain out to a range of $150 \mathrm{~km}$ from the radar. This study uses S-PolKa data from the DYNAMO legacy dataset (http://dynamo.ml-ext.ucar.edu/dynamo_legacy/), gridded to $0.5 \mathrm{~km} \times 0.5 \mathrm{~km} \times 0.5 \mathrm{~km}$ horizontal and vertical resolution for feature and rainfall analyses. Further details on quality control of the S-PolKa data are available from Dolan et al. (2017). Rain rates are estimated using S-PolKa's S-band reflectivity, differential reflectivity, and specific differential phase following algorithms described in Thompson et al. $(2015,2018)$ and calculated at $2.5-\mathrm{km}$ height.

\section{b. Echo-type classification}

The S-PolKa echoes are separated into convective and stratiform according to Powell et al. (2016). Expanding upon the common partitioning algorithm of Steiner et al. (1995), the amplitude and spatial extent of radar reflectivity in native polar coordinates are used to classify raining echo into six types: deep convection, stratiform, uncertain/mixed (deep convection and/or stratiform), isolated convective core, isolated convective fringe, and weak rain, where absolute reflectivity thresholds and values relative to background reflectivities distinguish these categories. A minimum areal coverage of $6 \mathrm{~km}^{2}$ is required for convective or stratiform echo classification. The "uncertain/mixed" category signals echoes adjacent to deep convective cores having heating profiles representative of both convection and stratiform precipitation and will be referred to as "mixed" for the remainder of the paper. For this study, as in Sakaeda et al. (2018), isolated convective core and fringe categories are combined because of their similar diurnal cycle in rainfall, and weak echoes are ignored because of their low $(<1 \%)$ contribution to total rain. 
To place our study into the context of ZH13 and explore the extreme end of the cloud population, convective echoes have been characterized into five types of three-dimensional echo objects: shallow isolated echoes (SCEs), deep convective cores (DCCs), wide convective cores (WCCs), deep and wide convective cores (DWCCs), and broad stratiform regions (BSRs). In general, SCEs represent small precipitating clouds dominated by warm-rain processes with echo tops more than $1 \mathrm{~km}$ below the $0^{\circ} \mathrm{C}$ level. DCCs indicate vigorous (generally young) convective cells with strong updrafts, defined by $30-\mathrm{dB} Z$ echo reaching $8 \mathrm{~km}$ in height. WCCs are often parts of intensifying stages of MCS development where active cells merge together, defined by a contiguous $30-\mathrm{dB} Z$ echo extending horizontally over $800 \mathrm{~km}^{2}$, and DWCCs are intense echoes that meet both the DCC and WCC criteria. BSRs are contiguous stratiform echoes exceeding $30000 \mathrm{~km}^{2}$ in the horizontal dimension. These echo definitions are modifications of thresholds applied extensively to the Tropical Rainfall Measuring Mission (TRMM) Precipitation Radar (PR) echo (e.g., Houze et al. 2007; Romatschke and Houze 2010; Houze et al. 2015) for examining tropical convection. The modifications occur for the horizontal spatial threshold of BSRs to account for the more limited ground-based radar spatial range, and we apply the "moderate" reflectivity thresholds (Houze et al. 2015) for oceanic convective systems. In addition to differences in the size and reflectivity thresholds, an important difference between this method and the Powell et al. (2016) classifications is the lack of height requirements in Powell et al. (2016).

\section{c. Particle identification}

Dominant hydrometeor type is inferred using S-PolKa data via the fuzzy logic algorithm of Vivekanandan et al. (1999). An overall explanation of this particle identification (PID) method and descriptions of hydrometeor types from this algorithm are presented in Rowe and Houze (2014) and Barnes and Houze (2014). Categories of interest include light, moderate, and heavy rain (LR, MR, HR), graupel/rain (GR), graupel/rimed aggregates $(\mathrm{GH})$, wet snow/aggregates (WS), dry snow/aggregates (DS), nonoriented/irregular small ice (II), and horizontally oriented small ice crystals (OI). Here, we use the same tuned hydrometeor algorithm as in those studies to describe general microphysical characteristics of precipitating echo, both for the extreme categories of ZH13 and for convective and stratiform classifications from Powell et al. (2016).

\section{d. Time periods}

MJO suppressed and active MJO periods during DYNAMO are defined here as in Sakaeda et al. (2018) based on filtered OLR anomalies from $15^{\circ} \mathrm{N}$ to $15^{\circ} \mathrm{S}$ globally averaged over a $5^{\circ}$ box around S-PolKa: active periods are 20-30 October, 20 November-1 December, and 14-22 December, and MJO suppressed periods are 3-14 October, 4-16 November, 5-9 December, and 26 December-8 January. REs are the 2-4-day periods of major rainfall identified by ZH13, defined by significant rain accumulation detected by S-PolKa. Figure 1 shows time series of hourly S-PolKa domain-accumulated rainfall (normalized by total number of S-PolKa files for that hour) for total (black), deep convective (red), and stratiform (blue) rain, with the 2-4-day REs of ZH13 highlighted in blue. Horizontal blue lines indicate the active periods of Sakaeda et al. (2018) showing that those active periods contain only 7 of the 11 REs identified by ZH13. The remaining four radar-based REs occurred either immediately before (Figs. 1a and 1b) or immediately after (Fig. 1c) the OLR-based active periods. Here, it is clear that the ZH13 REs do not extend over an entire MJO active period but are intermittent, concentrated periods of deep and mesoscale convection leading to high rainfall accumulations. Times considered part of active MJO periods that are not part of REs (i.e., the horizontal blue lines outside the blue shaded times) are considered the NE periods, which do contain rainfall but not of the same magnitude as during the REs. In this paper, we analyze the NE and RE times within active periods separately to investigate diurnal variability of rainfall, echo type, and microphysical characteristics during the times when most of the MJO rainfall was occurring.

\section{The diurnal cycle of precipitating echo during DYNAMO}

ZH13 examined the echo categories defined in section 2 (viz., SCEs, DCCs, DWCCs, WCCs, BSRs) for the 24-day REs during DYNAMO. We have calculated these categories for the entire DYNAMO S-PolKa dataset. Table 1 shows the total number of echo features stratified by type and month, highlighting the dominance of isolated shallow echo through the DYNAMO time period over the Indian Ocean, as previously noted by Barnes and Houze (2013). Deep convection (in the form of DCCs and DWCCs) was also frequently present. Deep convection that had grown upscale to larger (i.e., mesoscale) horizontal dimension (in the form of BSRs) occurred less often, but their rain-producing impact was large because of their size (Houze 1982, 2018). This table 

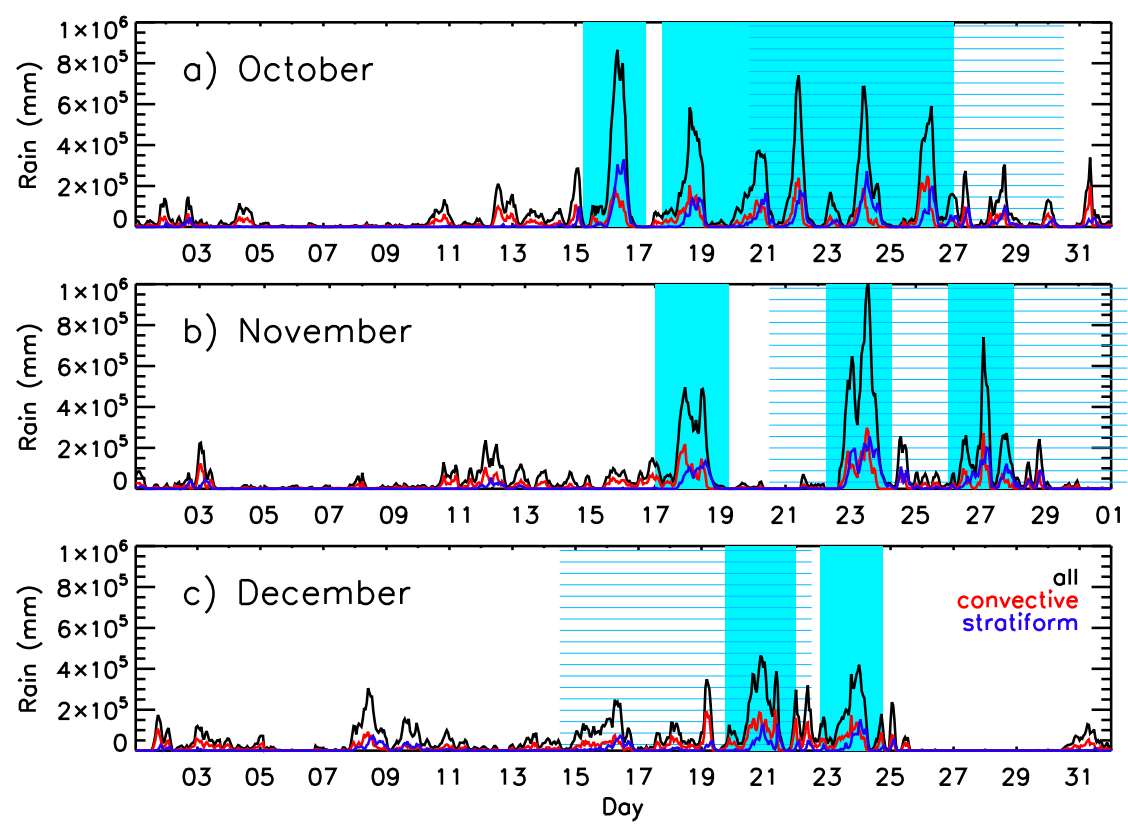

FIG. 1. Hourly S-PolKa domain-accumulated rainfall normalized by the total number of S-PolKa files per hour for (a) October, (b) November, and (c) December 2011. Rain is presented as all (black), convective (red), and stratiform (blue) as defined by Powell et al. (2016). Shaded blue regions indicate 2-day rain events as defined in ZH13. Blue horizontal lines indicate MJO active periods as defined in Sakaeda et al. (2018).

highlights differences between October, November, and December, each of which experienced an MJO passage (e.g., Gottschalck et al. 2013). While each month had similarly high numbers of shallow and deep echoes, December had noticeably fewer spatially larger convective echoes (DWCCs and WCCs) and no occurrences of BSRs, consistent with differences in echo structure for December described in Powell and Houze (2013) and Rowe and Houze (2014). Owing to the significantly fewer extreme echoes observed during January, we will focus the remainder of the study on October, November, and December.

The diurnal cycle of the echo objects presented in Table 1 is shown for each category for the entire October-December DYNAMO S-PolKa dataset (Fig. 2), with the total number of echo objects provided in parentheses. Plotted is a 5-h running average of the number of occurrences of a given echo type at a given hour of the day divided by the total number of occurrences for all hours. The frequently occurring SCEs did not exhibit a strong diurnal cycle; only a slight relative minimum at $0800 \mathrm{LT}$ is seen with a slight increase into the overnight hours. DCCs also had a minimum during the morning (0700-0800 LT), with a gradual increase into the evening and overnight hours peaking around local midnight. The diurnal evolution of DWCCs and WCCs was offset to a later time than DCCs, suggesting that mesoscale grouping of strong convection occurs at night and into the morning. The extension of WCCs into the afternoon and the peak of BSRs in late afternoon might appear surprising given that other studies in tropical oceanic regions (e.g., Romatschke et al. 2010; Zuluaga and Houze 2015; Sakaeda et al. 2018) have noted peak overall rainfall in the morning. This result suggests that while overall rain may be greater in the morning, the larger features, which are more likely to have top-heavy heating profiles, occurred later in the day in the S-PolKa domain. Sakaeda et al. (2018) found that the diurnal cycle over the entire period of DYNAMO, including both enhanced and suppressed MJO phases, had no clear trend for stratiform echo to maximize in the afternoon. However, they included all stratiform precipitation, whereas the BSR definition requires a large areal threshold, which is not required by the Powell et al. (2016) stratiform identification

TABLE 1. Total number of features identified using S-PolKa data for each month of DYNAMO.

\begin{tabular}{lrrcrr}
\hline & DCC & WCC & DWCC & BSR & SCE \\
\hline Oct 2011 & 1654 & 317 & 164 & 79 & 1780 \\
Nov 2011 & 1756 & 217 & 128 & 49 & 1793 \\
Dec 2011 & 1915 & 94 & 90 & 0 & 2030 \\
Jan 2012 & 167 & 18 & 14 & 0 & 605 \\
\hline
\end{tabular}




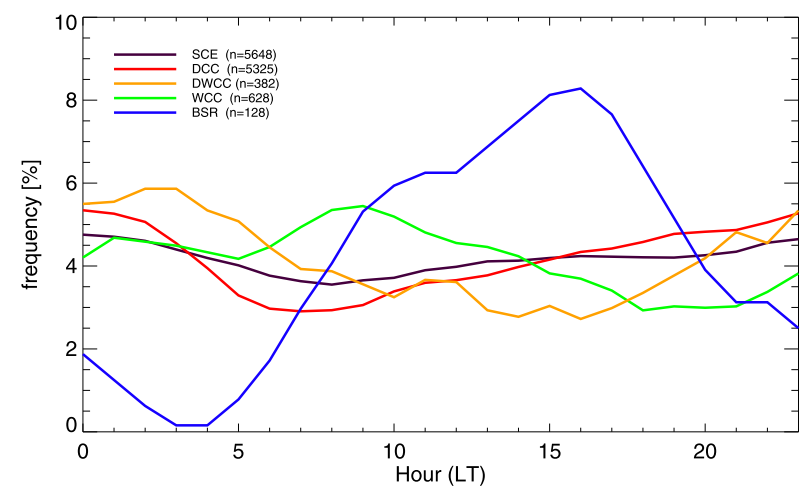

FIG. 2. Frequency of occurrence (\%) of each echo type as a function of local time as seen by the S-PolKa radar at Addu Atoll during the period October-December 2011. The frequency is the number of occurrences at a given time divided by the total number of occurrences over all hours during October-December. The total number of occurrences of each echo type is shown in parentheses. A 5-h running mean has been applied to smooth the data.

algorithm. We thus have identified only the most extensive stratiform echoes. The many smaller stratiform echoes identified by the Powell et al. (2016) stratiform method used in Sakaeda et al. (2018) probably includes simple fallout of convectively generated precipitation in the fringes of active convective zones, whereas the larger BSRs are those features most closely related to mature MCSs and thus most likely to have long-lasting potential vorticity generation in midlevels via a topheavy heating profile (Fritsch et al. 1986; Yang et al. 2017). That is, they are the features most likely to be providing dynamic feedback to larger scales of motion.

To explore the robustness of our results, we examine TRMM PR data within a grid box centered on S-PolKa and bounded by $3^{\circ} \mathrm{S}-3^{\circ} \mathrm{N}, 68^{\circ}-78^{\circ} \mathrm{E}$. This region is the same as that examined by Powell and Houze (2015). The overall evolution of echo types should be similar to Fig. 2 if S-PolKa reasonably sampled these convective storms spatially and temporally. To reduce error due to TRMM's infrequent temporal sampling (about twice a day at different times of day), at least a 4-h average should be applied to the data (Negri et al. 2002). We therefore applied a 5 -h running mean to the frequency of occurrence of TRMM echo object categories to produce Fig. 3 (similar to the running mean applied to the S-PolKa data in Fig. 2). This plot, using 16 years of TRMM PR data for the months of October-December, shows a progression of DCC peaks followed by DWCC, WCC, then BSR peaks. While this relative progression of echo categories is similar to that in Fig. 2, the timing is shifted with BSRs in the TRMM data increasing overnight and decreasing in the morning compared to the unusual afternoon peak in BSRs observed by S-PolKa.

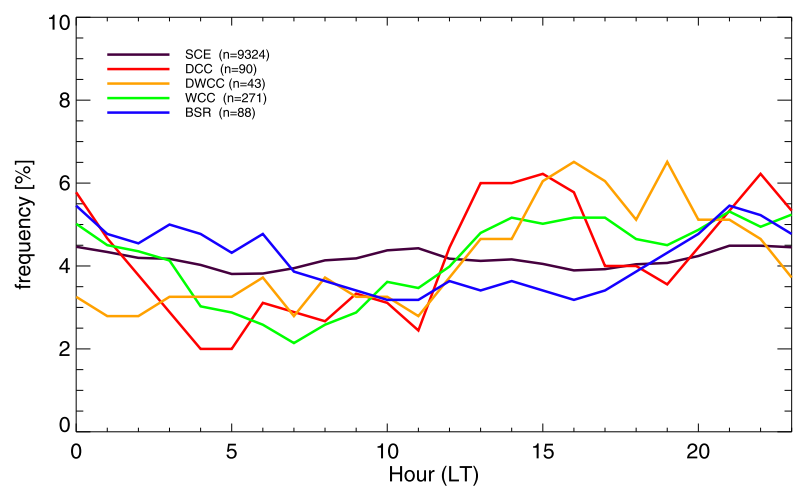

FIG. 3. As in Fig. 2, but for October-December over all 16 years of TRMM PR data.

While there are overall fewer samples of each category in the multiyear TRMM dataset compared to $\mathrm{S}-\mathrm{PolKa}$, it nevertheless appears from this comparison that the BSRs in the single year of DYNAMO may not be representative of a multiyear dataset. In addition, the multiyear dataset appears to have contained more MJO suppressed periods than MJO active periods compared to the DYNAMO period when the MJO was active during much of the campaign (e.g., Gottschalck et al. 2013). These inferences are consistent with the Sakaeda et al. (2018) result of a later morning rainfall peak during MJO active periods compared to suppressed periods being partly caused by greater frequency of upscale growth during those active periods. ZH13, however, noted that the episodic nature of rainfall, which was concentrated in $11 \mathrm{REs}$, occurred at various times of day during DYNAMO. Moreover, Gottschalck et al. (2013) found environmental differences between each MJO observed during the project. For these reasons, we will explore the diurnal cycle of precipitating echo in further detail by extracting S-PolKa echoes from each active MJO period (consisting of REs and NEs) in DYNAMO and comparing the statistics of those echoes to those only from the REs.

Figure 4 shows the mean number of extreme echo categories at each hour for all S-PolKa scans during that hour. Unlike the previous plots, we focus only on DCCs, DWCCs, and WCCs and divide the October-December DYNAMO time period into suppressed and active MJO periods, and the MJO active periods further into REs and NEs. Note that for REs, we show data for all 11 REs of ZH13 (REs all) and only those 7 that overlap with the Sakaeda et al. (2018) active MJO periods (REs active; see Fig. 1 for details). Error bars are also shown, representing the $95 \%$ confidence interval about the mean for each hour. Suppressed MJO periods contained DCCs, but generally lacked the categories associated with upscale growth of convective systems (i.e., DWCCs 

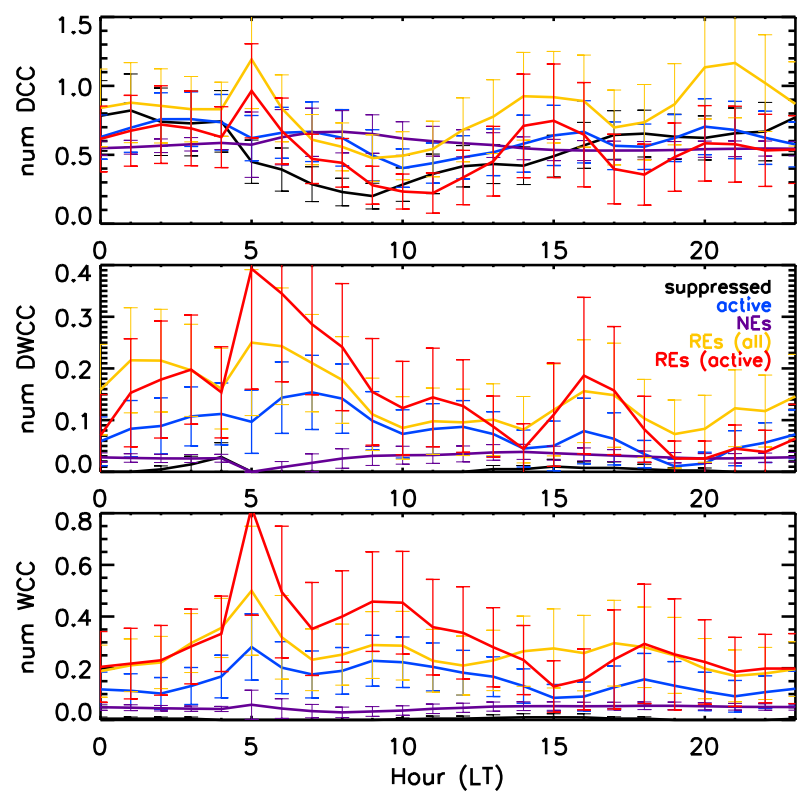

FIG. 4. Hourly mean time series (LT) of number of (top) DCCs, (middle) DWCCs, and (bottom) WCCs from S-PolKa scans at each hour. Individual lines represent time periods as follows: suppressed and active MJO periods as defined in Sakaeda et al. (2018), NEs defined as non-RE periods during active periods, all 11 REs from ZH13, and only the 7 REs corresponding to the defined active MJO periods. Error bars represent the $95 \%$ confidence intervals.

and WCCs). Active MJO periods do not show a clear diurnal cycle in DCCs and have a broad statistically significant peak in WCCs during late morning compared to early evening, but with WCCs observed at any hour of the day. These active periods were made up of REs and NEs that have different peak timing of extreme echo categories. DCCs in NEs followed the overall active period time series with the exception of around 1400 LT when the number of DCCs during REs increase, albeit with considerable statistical overlap with overall active periods. Similarly, where the active MJO time series (dark blue) and NE (purple) time series statistically differ most for DWCCs and WCCs were during periods with greater frequency of those echo types during REs (e.g., overnight into the morning). Therefore, focusing solely on the active periods as a whole misses differences between the timing of extreme echo during NEs versus REs that made up the active periods. The REs show statistically preferential times for DCCs, DWCC, and WCC categories in the early morning hours (0500 LT) with peaks that are significantly different (at the $95 \%$ confidence level) than during active periods. A secondary peak in DWCCs and WCCs in the afternoon is present during REs that is not present for NEs that warrants further analysis.
We further examine the diurnal cycle of DYNAMO convection through analysis of the Powell et al. (2016) echo-type categories to compare to the diurnal cycle of the more extreme categories showcased above. Specifically, and importantly, we investigate their rainfall contribution during the periods shown in Fig. 4. Figure 5 shows mean S-PolKa domain-accumulated rainfall for that hour and time period (e.g., suppressed versus active) for various rain types, with error bars again representing $95 \%$ confidence intervals. For all rain categories, the least amount of rain was produced during suppressed periods, with rainfall increasing in the late morning to a broad peak in convective rainfall following the diurnal variation in SSTs as described in Ruppert and Johnson (2015). Slightly more rain occurred during the NEs of the active periods compared to the suppressed. During these relatively quieter periods of the active periods, there was no clear diurnal cycle in the overall rain, as it was composed of convective rain that broadly peaked around $1100 \mathrm{LT}$ followed by mixed then stratiform rain that persisted into the afternoon. Active MJO periods overall contained more rain than the NEs contained within and had a slight tendency for more rain (all categories) to occur overnight, but with considerable spread, especially for stratiform rain. An even more pronounced amplitude in the diurnal cycle occurred during the REs. A statistically significant (at the 95\% confidence interval) overnight peak in deep convection for REs occurred at nearly the same time as mixed and stratiform categories, with all categories also producing more rain during the afternoon than during NEs and suppressed periods. This analysis therefore shows us the following:

- suppressed and active periods have different diurnal cycles in rainfall,

- NEs and REs have different diurnal cycles in rainfall during active periods, neither of which follow a similar cycle to suppressed MJO periods, and

- REs have statistically significant peak rainfall in the morning but also have peaks in rainfall later in the day both for deep convective and stratiform rainfall.

What this figure does not show is how these patterns may vary between the three individual MJOs observed during DYNAMO.

Table 2 shows the relative contribution to total rainfall from the REs for the whole DYNAMO period and during each active period. Note that each active period coincides with a calendar month so the active periods will be referred to as October, November, and December (see section 2 and Fig. 1 for exact dates of each active period). The 7 REs that occurred within the active periods are the focus of these rainfall comparisons, but all 

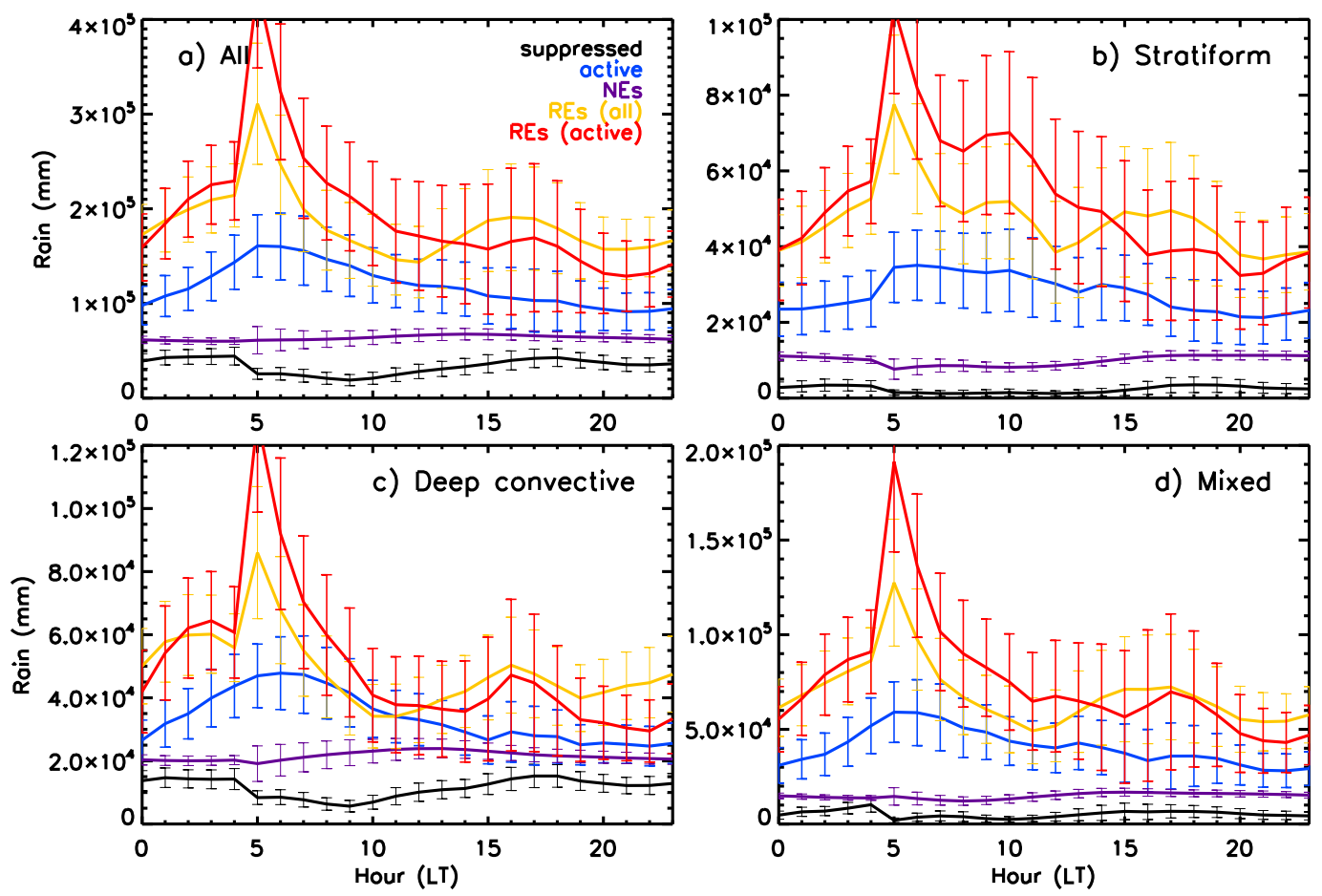

FIG. 5. Hourly time series (LT) of mean S-PolKa domain-accumulated rainfall at each hour for (a) all rain, (b) stratiform rain, (c) deep convective rain, and (d) mixed rain as defined in Powell et al. (2016). Time periods are defined as in Fig. 4. Error bars represent the $95 \%$ confidence intervals.

11 of the REs defined in ZH13 are also included (in parentheses). Overall, REs contributed to nearly half (three-quarters) of all stratiform rain observed by S-PolKa during active periods (during all ZH13 REs), with the majority of that $49 \%$ contribution occurring during the October and November REs. Note that the majority of the REs occurred during October, with only 1 of the 7 active-period REs occurring in December (or 2 of 11 if considering all REs). When comparing only to stratiform rain occurring during the individual MJOs, the REs accounted for $87 \%, 86 \%$, and $48 \%$ of all stratiform rain during the October, November, and December MJO active periods, respectively. Similarly, high percentages of deep convective rainfall in an individual active period came from the REs, again with the exception of December. The large-scale shear was different in December, leading to mesoscale systems taking the form of squall lines rapidly moving across the domain with less overall stratiform (e.g., Gottschalck et al. 2013; Powell and Houze 2013; Rowe and Houze 2014). Figure 1 also shows that a number of these squall-linetype events dominated by convection occurred within the active December MJO period outside of the REs.

The outlier of the three active MJO periods in DYNAMO, December, has been described in previous DYNAMO studies (e.g., Gottschalck et al. 2013) noting that this December period displayed a less coherent MJO signal than the previous two active periods and was characterized by strong westerlies. In contrast, easterly wind anomalies were observed across the domain for much of October and November, with the strongest wind anomalies corresponding to the transition from MJO suppressed to active periods during October and November. While it is clear from the table that REs during October and November accounted for the majority of the convective and stratiform rainfall during the October and November active periods, it is not clear if they followed a similar diurnal cycle. To investigate the diurnal cycle in all accumulated rainfall for each MJO, Fig. 6 shows the hourly mean time series of domainaccumulated S-PolKa rain for suppressed and active

TABLE 2. Fraction (\%) of convective and stratiform precipitation seen by the S-PolKa in DYNAMO that occurred during the 7 REs in the active periods, with all 11 of ZH13 REs in parentheses.

\begin{tabular}{lcccc}
\hline \hline & All REs & Oct REs & Nov REs & Dec REs \\
\hline All stratiform & $49(76)$ & $21(37)$ & $24(31)$ & $5(9)$ \\
Active stratiform & - & 87 & 86 & 48 \\
All convective & $32(52)$ & $14(24)$ & $11(17)$ & $7(12)$ \\
Active convective & - & 85 & 79 & 37 \\
\hline
\end{tabular}



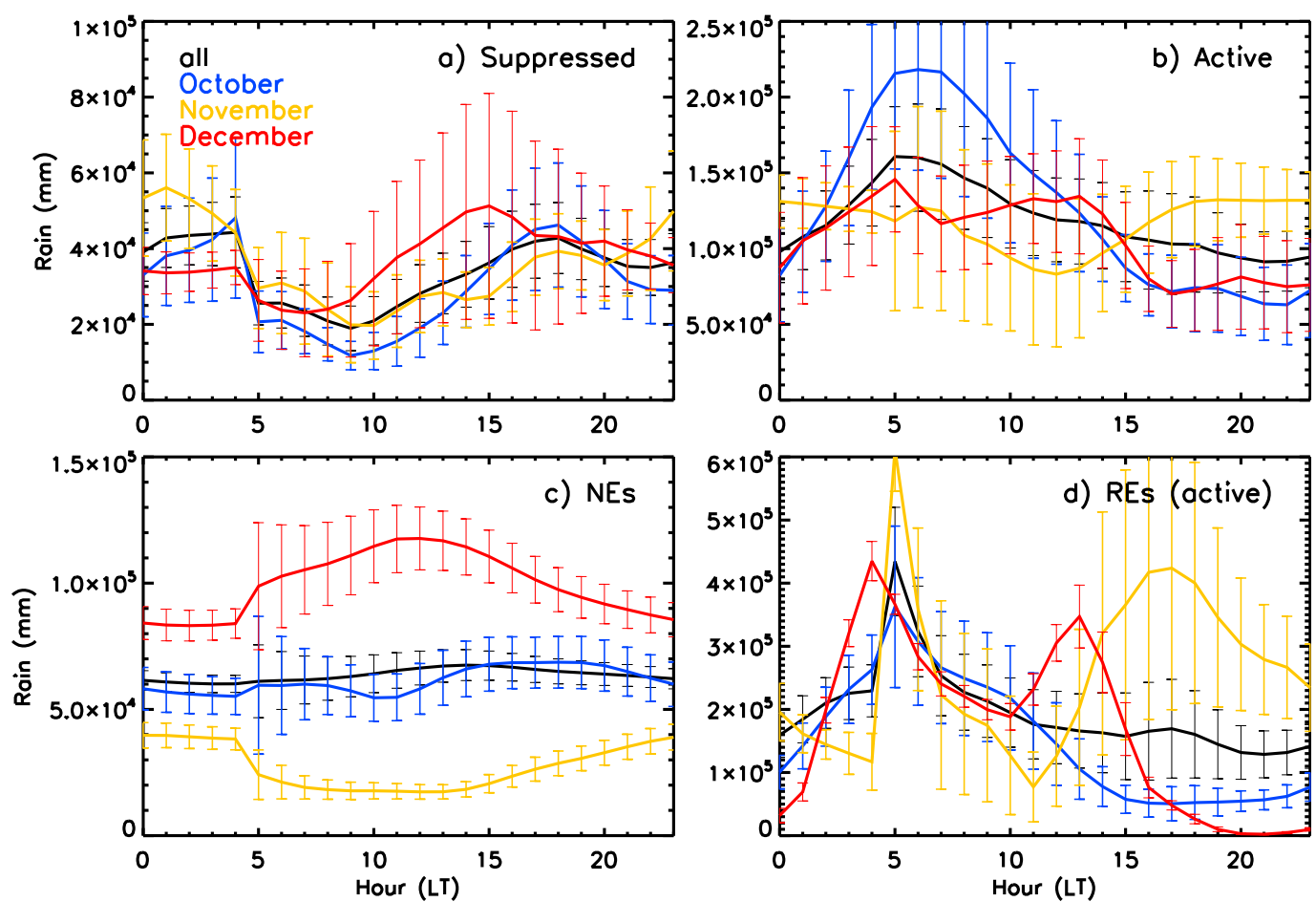

FIG. 6. Mean S-PolKa domain-accumulated rainfall for each hour (LT) and time period. Time series are divided into each month, showing rainfall from (a) MJO suppressed, (b) MJO active, (c) NE, and (d) RE periods. Error bars represent the $95 \%$ confidence intervals.

MJO periods (top) and for NEs and REs during active periods (bottom). Error bars again show $95 \%$ confidence intervals. Each time period is broken down into the individual month for comparison. MJO suppressed periods, as previously described in Rowe and Houze (2015), for example, exhibited an increase in rainfall throughout the day with a statistically significant peak in the afternoon and evening hours except for December. A larger spread is seen during December with an earlier peak in December from squall lines that moved through the domain. A later peak in November was dominated by the 11-12 November event (see Fig. 1). The suppressed diurnal cycle differed considerably from the active periods that overall had a rainfall peak overnight. However, as can be seen for the individual months, rainfall during individual active periods peaked at various times, with the overall time series reflecting the greater number of 2-day rainfall events during October that had a statistically significant peak in the early morning hours, while the November rainfall active periods had an overall much larger spread and statistically more rain during the afternoon (1600 LT) compared to October. December fell in between with squall lines moving through more during the early afternoon. When focusing on only the REs within the active periods, one would expect the overall pattern to closely follow the
October rainfall time series since four of the seven REs occur during October. While rainfall during the October REs had a statistically significant early morning peak, the overall RE (and for that matter, the overall active period) rainfall had a greater spread during the afternoon because of the large contribution to afternoon rainfall by the November REs. The intense raining period of 24-28 November, during which the two active REs occurred, was concentrated within two regions of eastward-propagating convection during a period of strong enhanced convective Kelvin wave activity (Gottschalck et al. 2013). Furthermore, Kerns and Chen (2018) found an important role of dry air intrusions associated with synoptic variability in the evolution of this MJO event that largely contributed to 1-2-day breaks in rainfall during the November active periods. During these breaks between the November REs, the NE rainfall had a statistically significant peak overnight, which differed between each month and from that during the REs.

Up to this point, the focus of the study has been on the diurnal cycle of precipitating echo both in terms of extreme echo categories (e.g., DCCs, WCCs, and DWCCs) and from all echo types (e.g., deep convection, stratiform). These analyses show that the diurnal progression of convection during REs, which accounted for the 

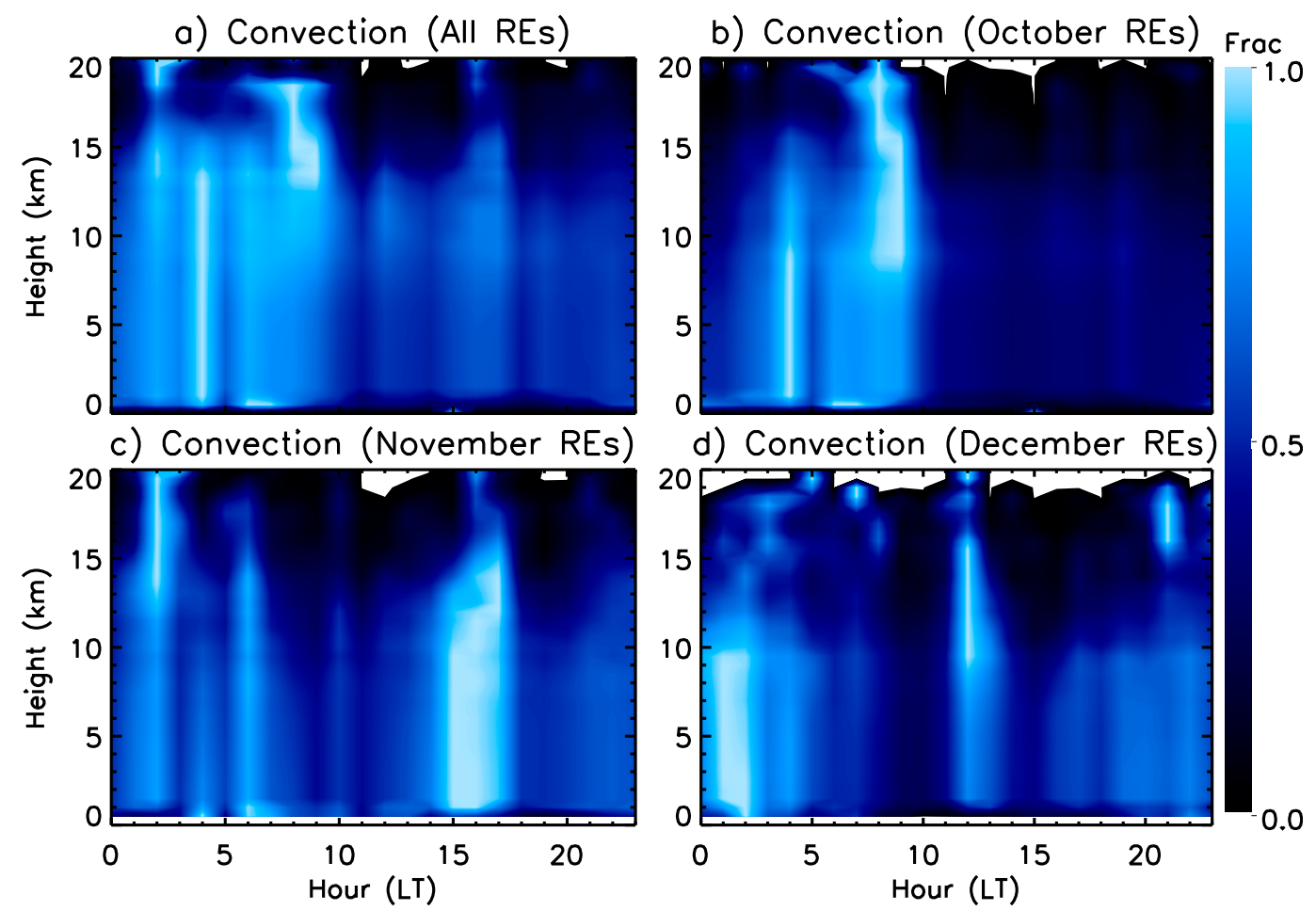

FIG. 7. Frequency contours of hydrometeors associated with deep convective rainfall with respect to hour (LT) during (a) all REs, (b) October REs, (c) November REs, and (d) December REs. Frequencies are normalized by the maximum frequency at each height and binned every $0.5 \mathrm{~km}$.

majority of the rainfall during the active MJO periods, was different from one MJO case to another. The peak local timing of convective and stratiform precipitation overall followed the statistically significant diurnal maximum in nocturnal rainfall during October (e.g., Yu et al. 2018), but was more strongly influenced by the synoptic-scale waves during November (e.g., Gottschalck et al. 2013). While this afternoon peak in November RE activity does not rule out a possible role of diurnal processes, it does emphasize the need for caution when reaching conclusions based on averages across multiple events.

\section{Microphysical evolution during DYNAMO rain events}

The analysis of the preceding section shows clear differences in $\mathrm{MJO}$ active periods in terms of rainfall but does not provide detail on the physical processes contributing to the observed rainfall patterns during the important, but relatively brief, REs when most of the rainfall occurs. Rowe and Houze (2014) noted deeper convection and a greater role of ice microphysics in MJO active periods relative to suppressed periods (as well as in October and November active periods compared to December), but it is not clear if the vertical structure of convection occurring within the MJO active periods differs depending on its timing within the diurnal cycle. For example, deep convection occurred during both the afternoon and overnight hours during each MJO active period and RE but peaked at different times for different active periods. To address our second science objective, that is, whether the microphysical characteristics within the echoes described above evolve in ways that indicate diurnal effects on precipitation during MJO REs, we use the PID algorithm described in section 2 within the context of different echo types.

We group together all hydrometeor categories (i.e., excluding all nonmeteorological categories) identified by the S-PolKa PID algorithm according to rain type. Here, we highlight results for deep convective (Fig. 7) and stratiform (Fig. 8) echo by plotting the number of S-PolKa grid points identified as any hydrometeor normalized by the maximum frequency of all hydrometeors at each height to see when the deepest echo occurred during REs. As was the case for accumulated rainfall, the hour of peak frequency of occurrence of PID varied for REs depending on the active period during which they occurred. Overall, hydrometeors associated with deep convection within REs peaked just prior to the 

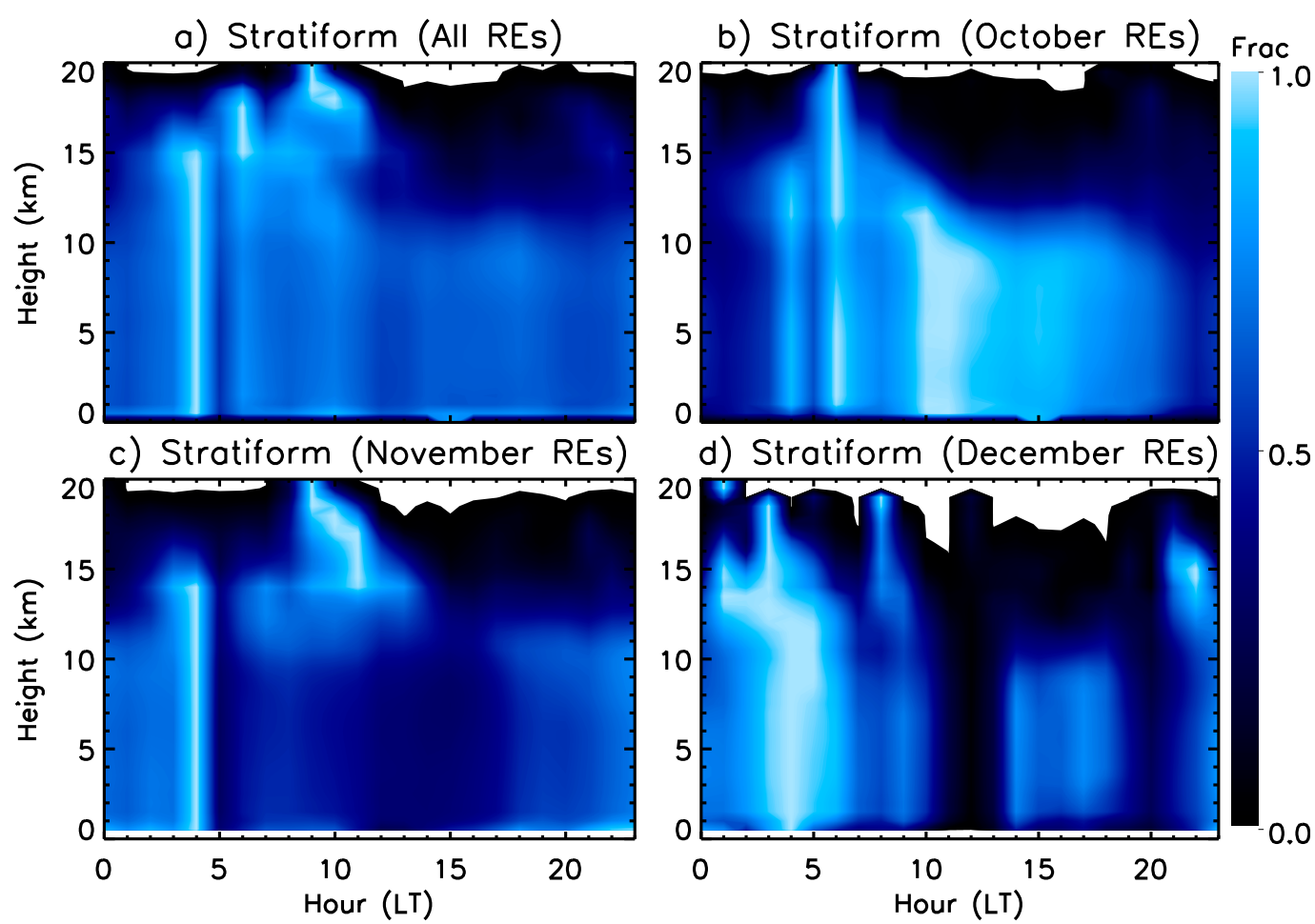

FIG. 8. As in Fig. 7, but for hydrometeors associated with stratiform rainfall.

peaks in rainfall (Fig. 6d). Deepest convective echo during the October REs occurred overnight into morning. During the November REs, rainfall peaked both overnight and in the afternoon (Fig. 6d). The deepest convective echo occurred overnight during the November REs, but overall the greatest frequencies of hydrometeors throughout most of the column occurred within a couple of hours leading up to the afternoon peak in accumulated rainfall (1600 LT). December had several periods of hydrometeors associated with deep convection, similar to several peaks in rainfall for the December RE (Fig. 6d). Hydrometeors associated with stratiform rainfall tended to reach the greatest heights overnight during periods of deep convection, corresponding to what we have seen in S-PolKa data during October and November-deep embedded convection decaying within broad stratiform regions (e.g., Powell and Houze 2013). When stratiform echo did occur during the afternoon, especially following the November $\mathrm{RE}$ afternoon rainfall maximum, stratiform echo tended to be shallower. Altogether, despite the differences in timing of REs during each active period, overall depth of echo was similar for a given echo-type category regardless of time of day. These results support the conclusion that convective activity in REs during active periods was dictated by factors in addition to the diurnal cycle. Hereafter, we focus on the microphysical evolution of precipitating systems during REs within a composite view of all REs.

Figure 9 shows the percentage of all hydrometeor categories at a given height and time relative to the total frequency of hydrometeors over all times at that height. This figure shows that the deepest echo preferentially occurred within a few hours prior to peak rainfall (hour $=0$ ) followed by a transition to shallower echo with time after the peak rainfall. There is also a bulge between $5 \mathrm{~km}$ (typical melting-level height) and $9 \mathrm{~km}$ of increased frequency for the hours between 4 and $12 \mathrm{~h}$, suggesting a shift to more stratiform-type echo with a marked brightband. This pattern corresponds to the ZH13 results showing a transition from peak occurrence of DCCs prior to peak rainfall with more concentration of hydrometeors in upper levels to wider and more stratiform echo after peak rainfall with midlevel concentration of hydrometeors. The total rainfall from each of the Powell et al. (2016) categories presented in this composite framework (Fig. 10) also show a shift in rainfall contribution from deep, isolated echo prior to peak rainfall transitioning to a greater contribution from stratiform rain after peak rainfall. Note that the peak rainfall coincided with the peak in rain from the mixed category, highlighting the important contribution to total rainfall from the transitioning convective-stratiform echo during REs. 


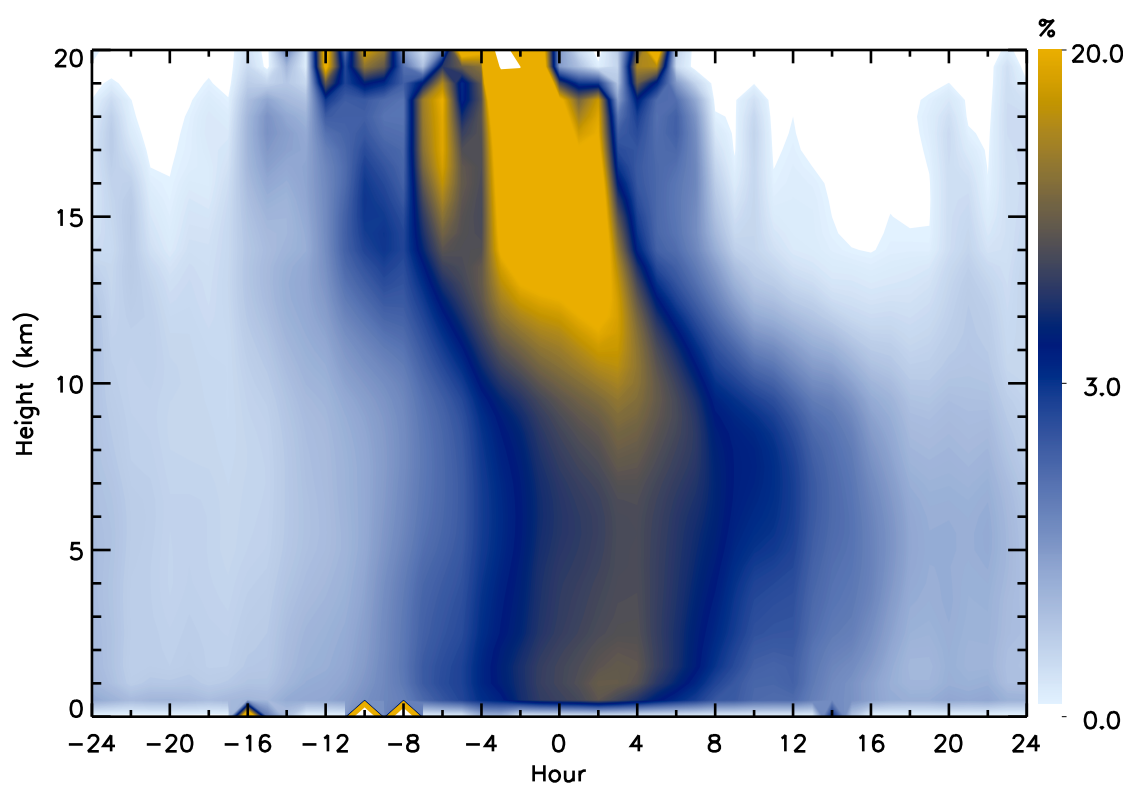

FIG. 9. Frequency of occurrence (\%) of hydrometeors composited over all REs. Hour 0 indicates time of peak rainfall. Contoured are percentages of hydrometeors at that hour relative to all hours within the 48 -h composites.

We use the above composites as a reference to compare the evolution of individual hydrometeor categories throughout the rain events. In Fig. 11, the frequency of occurrence of each PID category regardless of feature type (all grid points for the $11 \mathrm{REs}$ ) is plotted at each hour relative to the total frequency of each category for all $48 \mathrm{~h}$ of the composite. This framework does not provide the absolute frequency of occurrences of PID categories relative to each other but shows where individual categories peak relative to time of maximum rainfall. This figure highlights a transition from heavy rain increasing prior to maximum rain, with the maximum rain period coinciding with a shift from heavy to moderate rain, after which these rain categories drop off as the ice categories increase. After maximum rainfall, graupel, dry aggregates/snow, and wet/melting aggregates progressively peak in relative occurrence with wet aggregates and oriented ice reaching their relative maximum $4-8 \mathrm{~h}$ after peak rain. This progression in hydrometeor categories relative to maximum rainfall is consistent with the aforementioned transition from DCC to DWCC to WCC in ZH13 and our analysis of isolated, mixed, and stratiform echo. Heavy rain characterized DCCs and isolated convection, while moderate rain rates from deep convection and mixed echo (also meeting DWCC criteria) contributed most to peak rainfall. After peak rainfall, ice categories, especially wet aggregates, peaked, which are more associated with WCCs and stratiform echo.

\section{Conclusions}

Using ground-based dual-polarization radar (S-PolKa) data collected during the DYNAMO field campaign, this study has examined the diurnal cycle of oceanic convective systems during individual MJO events throughout the campaign. The goal of this analysis was to evaluate the influence of the diurnal cycle on convective systems during the rainfall events (REs) of MJO active periods to determine the relative importance of various physical scales (synoptic, diurnal, and microphysical) in determining RE precipitation, which dominates MJO rainfall. This study, placed in

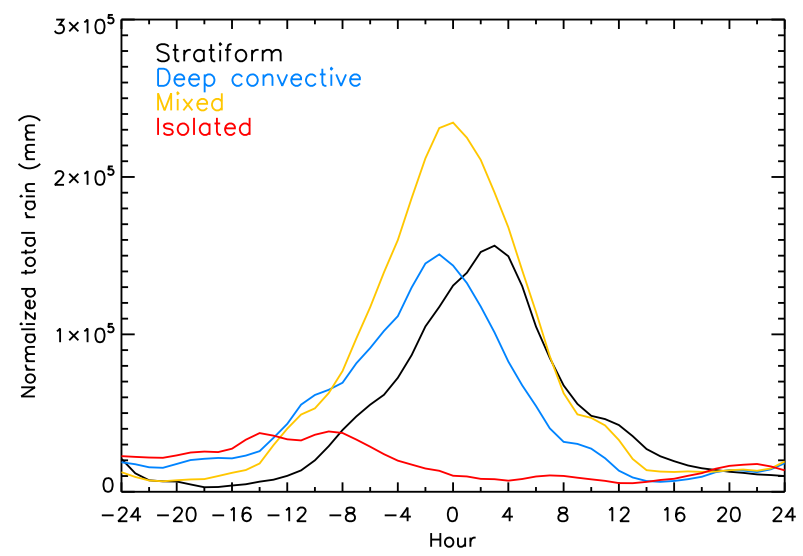

FIG. 10. Rainfall from each rain-type category composited over all REs with hour 0 representing time of maximum rainfall for each RE. Rain is normalized by the total number of files at that hour. 


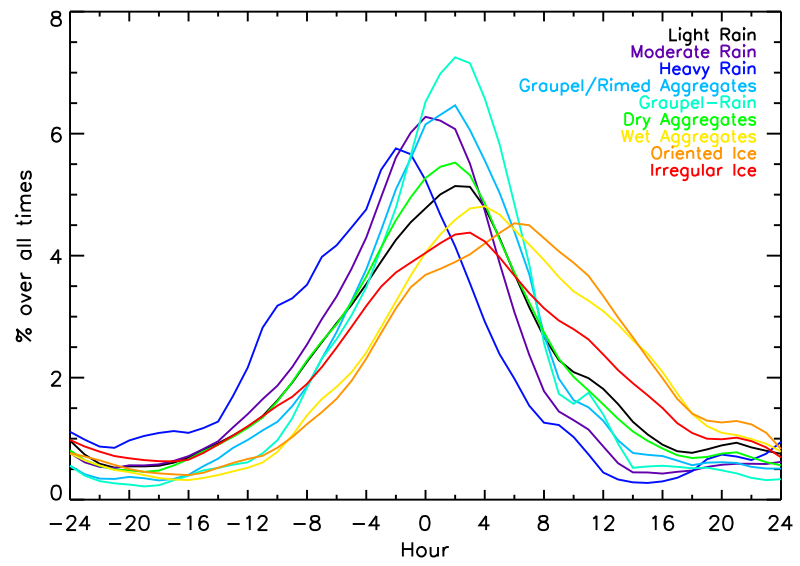

FIG. 11. Frequency of occurrence (\%) of each PID category for a given hour relative to all hours within the 48-h composites. Includes data from all 11 REs.

the context of previous work, points to an influence of synoptic-scale equatorial waves on the timing of major precipitation occurrence during MJO active periods. The diurnal cycle, which dominates the timing and evolution of systems during MJO suppressed periods, played a role during the quasi-2-day October periods, which featured statistically significant early morning peaks in rainfall, but the strong Kelvin wave activity during the November MJO strongly influenced the timing the convective systems leading to rainfall maxima from widespread echo (WCCs and stratiform) during the afternoon hours. This conclusion is especially apparent when focusing on occurrence of extreme echo types (e.g., DCCs, DWCCs, WCCs), which have a collective evolution over several days during active periods, as embodied in the Mapes et al. (2006) stretched building block notion.

Any two-way interaction between the convective population and large-scale influences (i.e., synopticscale waves embedded in the MJO) depends on how the vertical distribution of hydrometeors within the convective systems develops as the convection evolves within these wave-modulated rain events. ZH13 found that, statistically speaking, DCCs preceded maximum rainfall, followed by WCCs and BSRs for the 12-24 h after peak rain. They found this evolution of the gross radar echo statistics of the convection to be linked to the phase of parent synoptic-scale wave. We find that the microphysics active in the production of rain during the 2-4-day bursts of heavy precipitation have an important link to the phase of the parent synoptic-scale wave. Vertical distributions of hydrometeors shifted statistically from deep echo around the time of peak rainfall to maximum echo heights lower at $\sim 5-9-\mathrm{km}$ height in accordance with the phase of the synoptic wave. Composites of individual hydrometeor categories showed further that this evolving hydrometeor pattern was a result of wet/melting aggregates becoming more prevalent after maximum rainfall, following peaks in heavy and moderate rain and a reduction in the amount of dry snow aloft.

In connection with our science questions stated in the introduction, we therefore conclude that these results show that

- once a major precipitation event (RE) is triggered over the Indian Ocean during an active MJO period, convection grows upscale in concert with a synopticscale disturbance with varying degrees of influence of diurnally varying radiation and

- microphysical properties occurring in the 2-4-day rain events manifest statistically in concert with the synoptic time scales.

These results reinforce the idea that correct simulation of the MJO must take into account synoptic, diurnal, convective, and microphysical processes separately and distinctly in order to obtain the correct precipitation (i.e., energetic) characteristics of the MJO. It is especially important to recognize the intermittency of precipitation and diurnal processes, even within active phases of the MJO. As illustrated by this study, the occurrence of the rainfall and diurnal modulation of events differs for individual MJO rain events. By not recognizing this fact, a false impression of the diurnal cycle during active MJO periods is easily obtained. How this state of affairs affects simulation of the MJO and its downstream effects remains a challenge for future research and other investigators.

Acknowledgments. This research was supported by the National Science Foundation under Grant AGS-1355567. Zuluaga was supported with resources from Patrimonio Autónomo Fondo Nacional de Financiamiento para la Ciencia, La Tecnología y la Innovación, Francisco Jose de Caldas, and Universidad Nacional de Colombia. We appreciate the efforts of the NCAR S-PolKa team in the collection and quality control of the radar data. We also thank three reviewers for their constructive comments. Details on S-PolKa datasets are provided by the DYNAMO Legacy project (http://dynamo.ml-ext.ucar.edu/ dynamo_legacy/).

\section{REFERENCES}

Barnes, H. C., and R. A. Houze Jr., 2013: The precipitating cloud population of the Madden-Julian oscillation over the Indian and west Pacific Oceans. J. Geophys. Res. Atmos., 118, 69967023, https://doi.org/10.1002/jgrd.50375.

, and - 2014: Precipitation hydrometeor type relative to the mesoscale airflow in oceanic deep convection of the Madden-Julian oscillation. J. Geophys. Res. Atmos., 119, 13 990-14 014, https://doi.org/10.1002/2014JD022241. 
Chen, S. S., and R. A. Houze Jr., 1997: Diurnal variation and life cycle of deep convective systems over the tropical Pacific warm pool. Quart. J. Roy. Meteor. Soc., 123, 357-388, https:// doi.org/10.1002/qj.49712353806.

Dolan, B., P. Hein, S. Rutledge, and S. Powell, 2017: DYNAMO legacy rainfall products. UCAR, http://dynamo.ml-ext.ucar.edu/ dynamo_legacy/.

Feng, Z., S. Hagos, A. K. Rowe, C. D. Burleyson, M. N. Martini, and S. P. de Szoeke, 2015: Mechanisms of convective cloud organization by cold pools over tropical warm ocean during the AMIE/DYNAMO field campaign. J. Adv. Model. Earth Syst., 7, 357-381, https://doi.org/10.1002/ 2014MS000384.

Fritsch, J. M., R. J. Kane, and C. R. Chelius, 1986: The contribution of mesoscale convective weather systems to the warm-season precipitation in the United States. J. Climate Appl. Meteor., 25, 1333-1345, https://doi.org/10.1175/1520-0450(1986)025<1333: TCOMCW $>2.0 . \mathrm{CO} ; 2$.

Gottschalck, J., P. E. Roundy, C. J. Schreck III, A. Vintzileos, and C. Zhang, 2013: Large-scale atmospheric and oceanic conditions during the 2011-12 DYNAMO field campaign. Mon. Wea. Rev., 141, 4173-4196, https://doi.org/10.1175/ MWR-D-13-00022.1.

Houze, R. A., Jr., 1982: Cloud clusters and large-scale vertical motions in the tropics. J. Meteor. Soc. Japan, 60, 396-410, https://doi.org/10.2151/jmsj1965.60.1_396.

__, 2018: 100 years of research on mesoscale convective systems. A Century of Progress in Atmospheric and Related Sciences: Celebrating the American Meteorological Society Centennial, Meteor. Monogr., No. 59, Amer. Meteor. Soc., https://doi.org/10.1175/AMSMONOGRAPHSD-18-0001.1.

— D. C. Wilton, and B. F. Smull, 2007: Monsoon convection in the Himalayan region as seen by the TRMM Precipitation Radar. Quart. J. Roy. Meteor. Soc., 133, 1389-1411, https:// doi.org/10.1002/qj.106.

—, K. L. Rasmussen, M. D. Zuluaga, and S. R. Brodzik, 2015: The variable nature of convection in the tropics and subtropics: A legacy of 16 years of the Tropical Rainfall Measuring Mission (TRMM) satellite. Rev. Geophys., 53, 994-1021, https://doi.org/ 10.1002/2015RG000488.

Kerns, B. W., and S. S. Chen, 2018: Diurnal cycle of precipitation and cloud clusters in the MJO and ITCZ over the Indian Ocean. J. Geophys. Res. Atmos., 123, 10140-10161, https:// doi.org/10.1029/2018JD028589.

Liu, C., and M. W. Moncrieff, 1998: A numerical study of the diurnal cycle of tropical oceanic convection. J. Atmos. Sci., $\mathbf{5 5}$, 2329-2344, https://doi.org/10.1175/1520-0469(1998)055<2329: ANSOTD $>2.0 . \mathrm{CO} ; 2$

Madden, R. A., and P. R. Julian, 1971: Detection of a 40-50 day oscillation in the zonal wind in the tropical Pacific. J. Atmos. Sci., 28, 702-708, https://doi.org/10.1175/1520-0469(1971)028<0702: DOADOI $>2.0 . \mathrm{CO} ; 2$.

—_, and —_, 1972: Description of global-scale circulation cells in the tropics with a 40-50 day period. J. Atmos. Sci., 29, 1109-1123, https://doi.org/10.1175/1520-0469(1972)029<1109: DOGSCC $>2.0 . C O ; 2$.

Mapes, B. E., S. Tulich, J.-L. Lin, and P. Zuidema, 2006: The mesoscale convection life cycle: Building block or prototype for large-scale tropical waves? Dyn. Atmos. Oceans, 42, 3-29, https://doi.org/10.1016/j.dynatmoce.2006.03.003.

Negri, A. J., T. L. Bell, and L. Xu, 2002: Sampling of the diurnal cycle of precipitation using TRMM. J. Atmos. Oceanic
Technol., 19, 1333-1344, https://doi.org/10.1175/1520-0426(2002) 019<1333:SOTDCO $>2.0 . C O ; 2$.

Powell, S. W., and R. A. Houze Jr., 2013: The cloud population and onset of the Madden-Julian oscillation over the Indian Ocean during DYNAMO-AMIE. J. Geophys. Res. Atmos., 118, 11979-11 995, https://doi.org/10.1002/2013JD020421.

- , and - 2015: Evolution of precipitation and convective echo top heights observed by TRMM radar over the Indian Ocean during DYNAMO. J. Geophys. Res. Atmos., 120, 39063919, https://doi.org/10.1002/2014JD022934.

_ —_, and S. R. Brodzik, 2016: Rainfall-type categorization of radar echoes using polar coordinate reflectivity data. J. Atmos. Oceanic Technol., 33, 523-538, https://doi.org/10.1175/ JTECH-D-15-0135.1.

Randall, D., Harshvardhan, and D. A. Dazlich, 1991: Diurnal variability of the hydrologic cycle in a general circulation model. J. Atmos. Sci., 48, 40-62, https://doi.org/10.1175/1520-0469(1991) $048<0040$ :DVOTHC $>2.0 . \mathrm{CO} ; 2$.

Romatschke, U., and R. A. Houze Jr., 2010: Extreme summer convection in South America. J. Climate, 23, 3761-3791, https://doi.org/10.1175/2010JCLI3465.1.

— - S. Medina, and R. A. Houze Jr., 2010: Regional, seasonal, and diurnal variations of extreme convection in the South Asian region. J. Climate, 23, 419-439, https://doi.org/10.1175/ 2009JCLI3140.1.

Rowe, A. K., and R. A. Houze Jr., 2014: Microphysical characteristics of MJO convection over the Indian Ocean during DYNAMO. J. Geophys. Res. Atmos., 119, 2543-2554, https:// doi.org/10.1002/2013JD020799.

— the transition from suppressed to active MJO conditions. J. Geophys. Res. Atmos., 120, 10-324-10 350, https://doi.org/ 10.1002/2014JD022948.

Ruppert, J. H., and R. H. Johnson, 2015: Diurnally modulated cumulus moistening in the preonset stage of the Madden-Julian oscillation during DYNAMO. J. Atmos. Sci., 72, 1622-1647, https://doi.org/10.1175/JAS-D-14-0218.1.

Sakaeda, N., S. W. Powell, J. Dias, and G. N. Kiladis, 2018: The diurnal variability of precipitating cloud populations during DYNAMO. J. Atmos. Sci., 75, 1307-1326, https://doi.org/ 10.1175/JAS-D-17-0312.1.

Steiner, M., R. A. Houze Jr., and S. E. Yuter, 1995: Climatological characterization of three-dimensional storm structure from operational radar and rain gauge data. J. Appl. Meteor., 34, 1978-2007, https://doi.org/10.1175/1520-0450(1995)034<1978: CCOTDS $>2.0 . \mathrm{CO} ; 2$.

Thompson, E. J., S. A. Rutledge, B. Dolan, and M. Thurai, 2015: Drop size distributions and radar observations of convective and stratiform rain over the equatorial Indian and west Pacific Oceans. J. Atmos. Sci., 72, 4091-4125, https://doi.org/10.1175/ JAS-D-14-0206.1.

,,,,$-- \ldots$, and V. Chandrasekar, 2018: Dual-polarization radar rainfall estimation over tropical oceans. J. Appl. Meteor., 57, 755-775, https://doi.org/10.1175/JAMC-D-17-0160.1.

Vivekanandan, J., D. S. Zrnić, S. M. Ellis, R. Oye, A. V. Ryzhkov, and J. Straka, 1999: Cloud microphysics retrieval using S-band dualpolarization radar measurements. Bull. Amer. Meteor. Soc., 80, 381-388, https://doi.org/10.1175/1520-0477(1999)080<0381: CMRUSB $>2.0 . \mathrm{CO} ; 2$

Yang, Q., R. A. Houze Jr., L. R. Leung, and Z. Feng, 2017: Environments of long-lived mesoscale convective systems over the central United States. J. Geophys. Res. Atmos., 122, 13288 13 307, https://doi.org/10.1002/2017JD027033. 
Yoneyama, K., C. D. Zhang, and C. N. Long, 2013: Tracking pulses of the Madden-Julian oscillation. Bull. Amer. Meteor. Soc., 94, 1871-1891, https://doi.org/10.1175/BAMS-D12-00157.1.

Yu, H., R. H. Johnson, P. E. Ciesielski, and H.-C. Kuo, 2018: Observation of quasi-2-day convective disturbances in the equatorial Indian Ocean during DYNAMO.J. Atmos. Sci., 75, 2867-2888, https://doi.org/10.1175/JAS-D-17-0351.1.
Zuluaga, M. D., and R. A. Houze Jr., 2013: Evolution of the population of precipitating convective systems over the equatorial Indian Ocean in active phases of the Madden-Julian oscillation. J. Atmos. Sci., 70, 2713-2725, https://doi.org/10.1175/JAS-D-12-0311.1. , and - 2015: Extreme convection of the near-equatorial Americas, Africa, and adjoining oceans as seen by TRMM. Mon. Wea. Rev., 143, 298-316, https://doi.org/10.1175/MWRD-14-00109.1. 